2017-06-04

\title{
Estimation of Train Driver Workload: Extracting Taskload Measures from On-Train-Data-Recorders
}

\author{
Nora Balfe \\ Trinity College Dublin, Ireland \\ Katie Crowley \\ Trinity College Dublin, Ireland \\ Brendan Smith \\ larnród Éireann
}

See next page for additional authors

Follow this and additional works at: https://arrow.tudublin.ie/scschcomcon

Part of the Artificial Intelligence and Robotics Commons

\section{Recommended Citation}

Balfe N., Crowley K., Smith B., Longo L. (2017) Estimation of Train Driver Workload: Extracting Taskload Measures from On-Train-Data-Recorders. In: Longo L., Leva M. (eds) Human Mental Workload: Models and Applications. H-WORKLOAD 2017. Communications in Computer and Information Science, vol 726. Springer, Cham. DOI: 10.1007/978-3-319-61061-0_7

This Conference Paper is brought to you for free and open access by the School of Computer Sciences at ARROW@TU Dublin. It has been accepted for inclusion in Conference papers by an authorized administrator of ARROW@TU Dublin. For more information, please contact arrow.admin@tudublin.ie, aisling.coyne@tudublin.ie,gerard.connolly@tudublin.ie.

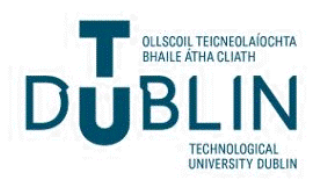




\section{Authors}

Nora Balfe, Katie Crowley, Brendan Smith, and Luca Longo

This conference paper is available at ARROW@TU Dublin: https://arrow.tudublin.ie/scschcomcon/342 
See discussions, stats, and author profiles for this publication at: https://www.researchgate.net/publication/318145213

\section{Estimation of Train Driver Workload: Extracting Taskload Measures from On- Train-Data-Recorders}

Conference Paper in Communications in Computer and Information Science · June 2017

DOI: 10.1007/978-3-319-61061-0_

CITATIONS

14

4 authors, including:

Nora Balfe

Trinity College Dublin

59 PUBLICATIONS 380 CITATIONS

SEE PROFILE

Luca Longo

Technological University Dublin - City Campus

90 PUBLICATIONS 1,090 CITATIONS

SEE PROFILE

\section{READS}

603

Katie Crowley

University of Limerick

19 PUBLICATIONS 223 CITATIONS

SEE PROFILE

Some of the authors of this publication are also working on these related projects:

IDENTIFICATION AND MEASUREMENT OF VARIABLES AFFECTING COMPONENT LIFETIME: A CASE STUDY OF POLYMER DEGRADATION IN THE BIOPHARMACEUTICAL INDUSTRY View project

Tosca: Total Safety Management for safety critical activities View project 


\title{
Estimation of Train Driver Workload: Extracting Taskload Measures from On-Train-Data-Recorders
}

\author{
Nora Balfe ${ }^{1}$, Katie Crowley ${ }^{1}$, Brendan $\mathrm{Smith}^{2}$ and Luca Longo ${ }^{3}$ \\ ${ }^{1}$ Trinity College Dublin, Dublin, Ireland \\ ${ }^{2}$ Iarnród Éireann, Dublin, Ireland \\ ${ }^{3}$ Dublin Institute of Technology, Dublin, Ireland
}

\begin{abstract}
This paper presents a method to extract train driver taskload from downloads of on-train-data-recorders (OTDR). OTDR are in widespread use for the purposes of condition monitoring of trains, but they may also have applications in operations monitoring and management. Evaluation of train driver workload is one such application. The paper describes the type of data held in OTDR recordings and how it can be transformed into driver actions throughout a journey. Example data from 16 commuter journeys is presented, which highlights the increased taskload during arrival at stations. Finally, the possibilities and limitations of the data are discussed.
\end{abstract}

Keywords: OTDR, train driver taskload, rail human factors

\section{Introduction}

In contrast to rail signalling, where several specific workload tools have been developed (e.g. [1]), train driver workload is under-researched. The train driver task has however been extensively discussed in the human factors literature, with numerous models, frameworks and task analyses produced to describe the task and influencers, and several studies investigating train driver visual behavior (e.g. [2,3]). This paper presents a new approach to investigating train driver workload using data from on-train-data recorders (OTDR) to capture train driver activity. This section describes the work to date on measurement of workload in train driving, and Section 2 proposes a new method for calculating train driver taskload from OTDR. Section 4 presents a preliminary application of the methodology in a case study of 16 commuter train journeys. Finally, the limitations, possible applications and further research required are discussed in Section 5.

Human factors research into the train driving task dates back to Branton [4], who in 1979 published a paper discussing the nature of train driving and the need for drivers to anticipate future actions, develop internal representations of the railway (route knowledge), and test these representations against reality. Authors who have written about the train-driving task typically agree that the key tasks involve processing information collected from inside and outside the cab and applying route 
knowledge to correctly control the speed and braking of the train [5.6.7]. Additional tasks include:

- Maintaining an efficient speed profile [5]

- Making scheduled stops [5]

- Managing the train for fuel efficiency [6]

- Departing stations [8]

- Arriving at stations [8]

Gillis [9] notes that the train driving task is primarily a visual-spatial task involving constant perception and processing of information, and the majority of train driver physical actions are driven by information received (e.g. moving the traction handle in response to a change in the speedometer). Hamilton \& Clarke [10] include a high level cognitive task analysis (CTA) goal structure, which was used as the basis of a quantitative tool for the assessment of route drivability (Table 1).

Table 1: CTA of the Train Driver Task [10]

\begin{tabular}{|c|c|c|}
\hline \multirow[t]{10}{*}{ Execute a train service } & \multirow[t]{3}{*}{ Prepare for service } & $\begin{array}{l}\text { Prepare driver for driving } \\
\text { duty }\end{array}$ \\
\hline & & Assemble train (shunting) \\
\hline & & Prepare train for service \\
\hline & \multirow[t]{5}{*}{ Drive service } & Start from scheduled stop \\
\hline & & $\begin{array}{l}\text { Drive towards scheduled } \\
\text { service stop in accordance } \\
\text { with movement authority }\end{array}$ \\
\hline & & $\begin{array}{l}\text { Stop for scheduled service } \\
\text { stop }\end{array}$ \\
\hline & & $\begin{array}{l}\text { Perform service operations } \\
\text { at stop }\end{array}$ \\
\hline & & $\begin{array}{l}\text { Perform operations for } \\
\text { failed train }\end{array}$ \\
\hline & \multirow[t]{2}{*}{$\begin{array}{l}\text { Close out train after } \\
\text { service }\end{array}$} & $\begin{array}{l}\text { Relinquish possession of } \\
\text { train }\end{array}$ \\
\hline & & $\begin{array}{l}\text { Perform formalities after } \\
\text { service }\end{array}$ \\
\hline
\end{tabular}

However, despite the apparent simplicity of the task, Naweed [11] describes the train driving task as complex, dynamic, and opaque. Although the basic tasks may be described reasonably simply, the actual practice involves changing conditions, event densities, and performance pressures that drive adjustments in motor skills and problem solving strategies. The complexity is driven by sometimes conflicting goals of time-accuracy, comfort and speed regulation and the trade-offs required to optimise the overall journey. The dynamism comes from the constant need to regulate speed and finally, the opacity is due to the gaps in information when working with lineside signalling. Drivers must use their route knowledge to infer future requirements. Thus, driver performance is not simply a matter of perceiving and responding to stimuli as suggested by the use of simple information processing models, but is driven by continuous, proactive prediction and planning [12]. The consensus in the literature is 
that, despite the apparent simplicity, train driving is a complex task requiring processing and integration of vestibular, kinaesthetic, acoustic, and peripheral vision information [13].

Naweed [11] describes a closed loop system of train driver performance based on the perception of location from lineside features, and use of this information in conjunction with the drivers' knowledge base (i.e. route knowledge and train handling knowledge) to establish location and apply appropriate controls (Figure 1). Driving strategies are informed by specific sources, including the rule book and temporary notices. Hamilton \& Clarke [10] describe how train-driving goals are selected by a plan (or rules) determined by operating conditions. For example, on passing a cautionary aspect a driver should decelerate, but when and by how much will depend on situational factors including the specific aspect shown, railhead conditions, train performance characteristics, etc.

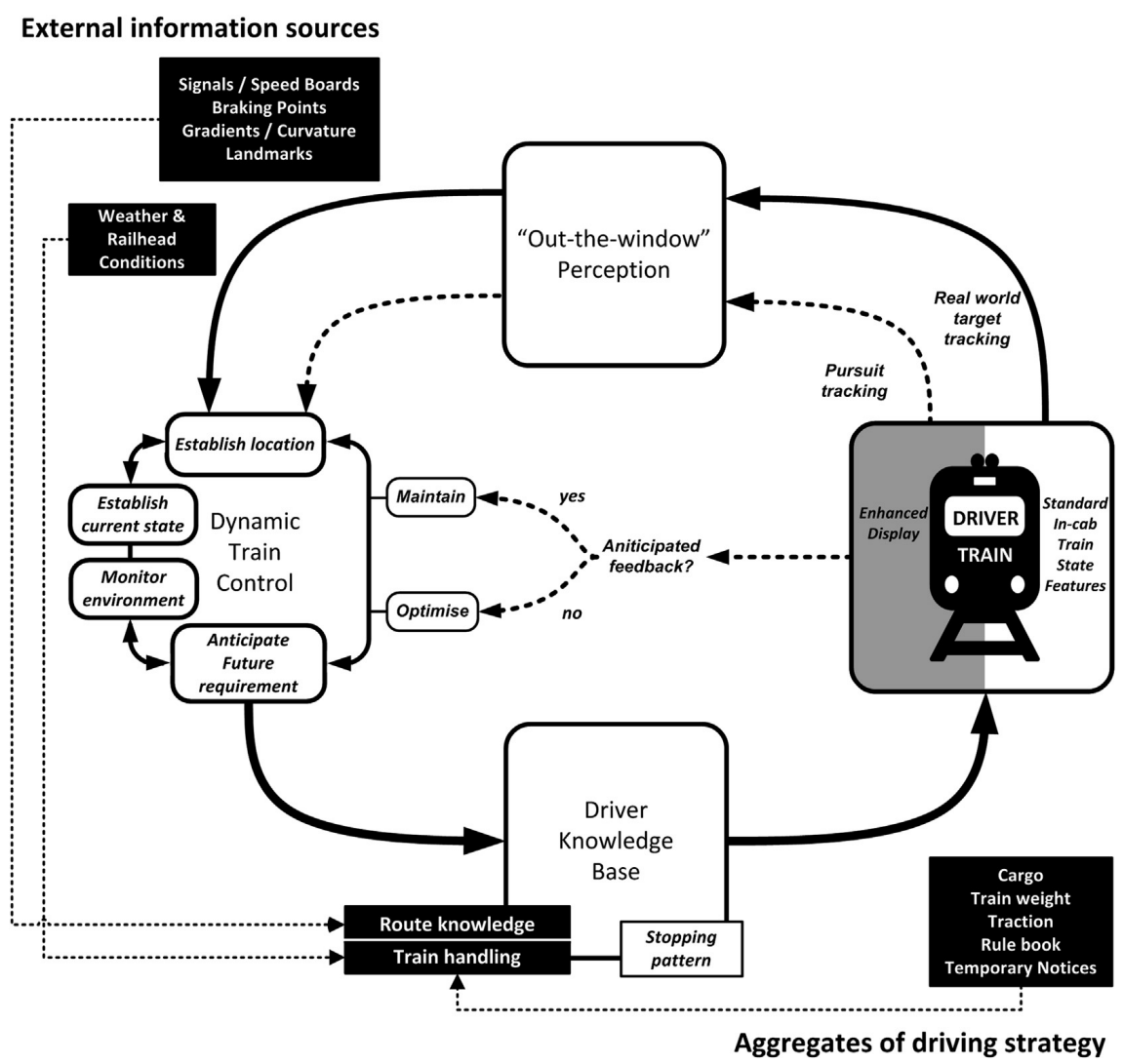

Figure 1: Model of the Train Driver Task [11]

These models acceptably describe moment-to-moment train control, but are less capable of representing the fullness of the train driving task, particularly its contextual and situated nature [14]. McLeod et al. [14] suggest instead a situational model of 
driver performance which applies the concept of situation awareness to link driver knowledge and experience with their actions and strategies (Figure 2).

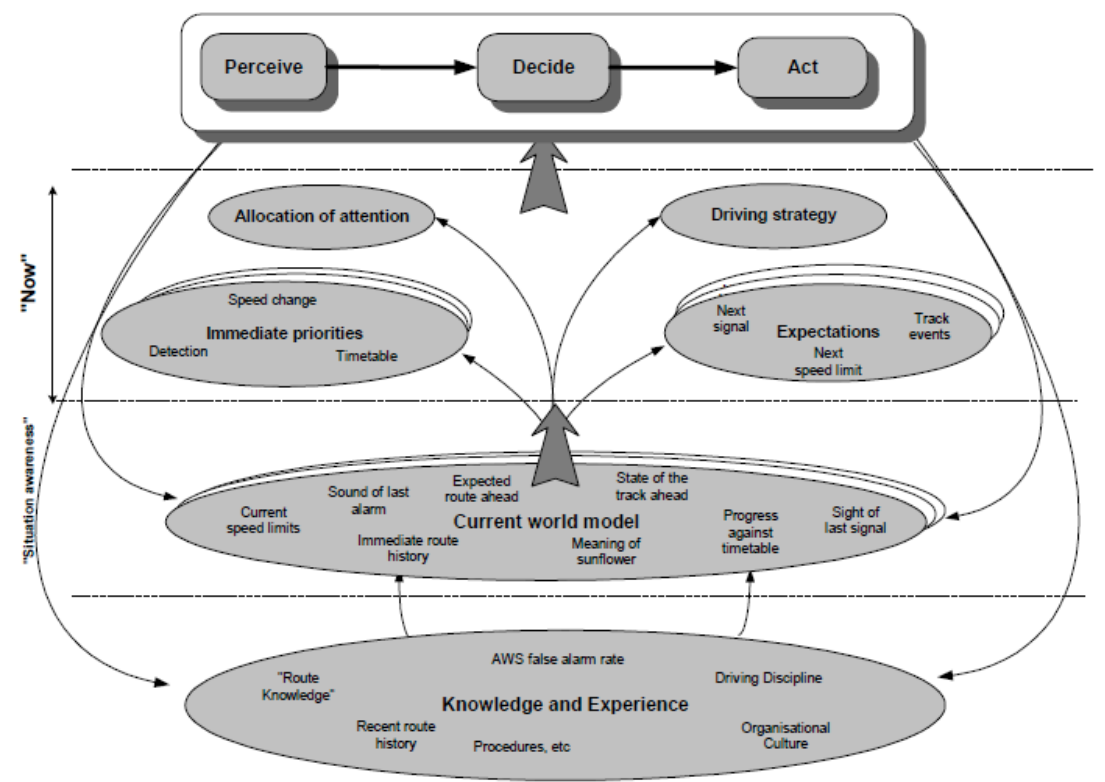

Figure 2: Situational model of the train driver performance [14]

In addition to this more complex model, McLeod et al. [14] discuss additional concepts that may be relevant to explore the complexity associated with the task:

- Strategic behaviour - how do train drivers develop and apply strategies for managing workload, attention, and other influences?

- Situation awareness - how do drivers develop an understanding of the current situation and apply this to predict future state?

- Situated behaviour and distributed cognition - how do the context, situation, tools and surroundings inform and support or hinder driver actions?

- Distributed cognition - how do the artefacts and surrounding environment support or hinder driver performance?

\subsection{Train Driver Workload Measurement}

Despite the number of models of train driving, measurement of train driver workload has been specifically investigated in only a small number of papers. Dunn and Williamson [15] examined the effect of underload on train driver performance in a simulated train-driving environment. They suggest that the train-driving task can involve periods of relatively high workload, but also involves "periods of repetitive low workload activity, such as driving along a straight track at a steady speed and 
only responding to signals from the in-cab 'vigilance' control device." (pp. 998). As train drivers do not control lateral positioning of the train (although they must be vigilant approaching junctions that they do not take the 'wrong route'), their driving tasks are limited to controlling the throttle and brake. Dunn and Williamson [15] also state that the train-driving environment itself may add to the experience of monotony "with drivers subjected to either the repetitive, unchanging stretches of train tracks moving beneath the train and off into the distance, or the reduced external stimuli when driving underground in a tunnel or at night" (pp. 998). They used self-report techniques (NASA-TLX) and primary task performance to investigate the differences in workload between a high and low monotony simulated train-driving task and found a detrimental effect of the combination of low task demands and monotony.

The widespread adoption of on-train-data-recorders (OTDR) offers a new approach to measuring and potentially monitoring train driver workload. OTDR are primarily used for train fault monitoring and management, but as they log each and every input in the train cab they may also have an application in monitoring train driver activities. Walker and Strathie [16] suggest that train recorder data is an underused but potentially important data source for understanding human performance and detecting risks in advance of accidents. Broekhoven [17] used real-time data from operational signalling control systems to calculate an External Cognitive Task Load (XTL) for rail signallers. The approach uses four measures over five minute periods: the number of automatically executed plan rules (monitoring load), the number of manually adjusted plan rules (planning load), the number of non-executed plan rules (manual intervention load), and the percentage of seconds spoken through the telephone (communications load). The four measures were weighted to align with the Integrated Workload Scale for Signallers [1] and then summed. This result was then multiplied by the a switching cost composed of the number of delayed train, the number of telephone calls and the number of incidents. The XTL formula was found to discriminate between high and low perceived workload in both the communication and manual actions.

The purpose of this paper is to describe a method of extracting train driver actions from the OTDR, and present the results of a case study describing train driver taskload as measured by the OTDR.

\section{Method}

\subsection{Experiment Description}

Data were collected from eight return journeys (16 journeys in total) over two routes. Five drivers participated in the study. Table 1 describes the Driver and route for each journey included in the study. A researcher travelled with the driver on each journey, and collected subjective and physiological data; this data will not be presented in this paper. The journeys were all scheduled passenger services, and the research did not require any changes to the timetabled journey. 


\subsection{Dataset Preparation}

Following the journey, the OTDR data were downloaded from the Teloc (Hasler, V3.11) via the Nexala remote condition monitoring system (Nexala, v2.8.01). The resulting Teloc files were parsed, cropped to the relevant timeframe, relevant signals selected, and exported to Excel using Eva 2 software (Hasler, v2.4 Pro). The following signals were available and regarded as relevant as they are directly attributable to driver actions:

- Brake demand - provided in three bitcodes

- Acknowledgement of CAWS warning system

- Aspect logged in the CAWS system (Green, Yellow, Double Yellow, Red)

- Gear (forward/reverse)

- Emergency brake application

- Headlight (dipped beam and full beam)

- Horn switch

- Left door opening

- Power demand - provided in three bitcodes

- Right door opening

- Vigilance alarm acknowledgement

All these signals were logged as bitcodes (0/1); in addition, analogue signals of time, speed, and GPS coordinates were downloaded and exported for each journey.

The data was then pre-processed via the following steps:

1. Power and brake levels applied were determined from the relevant bitcodes for each line of data;

2. The aspect (signal colour) was determined for each line of data;

3. Journey phases were added according to the framework described by Balfe \& Smith [18];

This dataset provided the basis for the analysis of train driver actions or taskload.

\subsection{Driver Taskload Computation}

The dataset was used to calculate driver taskload by identifying the times of driver actions. The actions identifiable from the data are:

- Initiate braking - Drivers must use their route knowledge and timetable knowledge to identify when they should start applying the brakes for the next station stop, red signal stop, or to reduce or control speed;

- Change braking - Drivers adjust the level of braking according to the train and braking performance;

- Stop braking - Drivers remove brakes when they no longer wish to reduce train speed, or the train is stopped; 
- Change gear - Drivers may put the train into reverse - this is unusual during a normal passenger journey and would usually be performed in shunting or permissive working (e.g. separating previously joined trains) movements;

- Acknowledge CAWS warning - Drivers receive a buzzer warning when they approach a more restrictive aspect, and they must acknowledge this warning by pressing a button within 7 seconds, or the train emergency brake will be automatically applied;

- Headlights - Drivers change headlight settings as they move through the network;

- Horn switch - Drivers operate the train horn at required locations on the network, and often as they enter or leave a station;

- Door opening - Drivers operate the door switches to open and close the train doors at stations. There are several unlogged tasks associated with closing train doors - specifically checking for passengers trapped in doors and checking that the door interlock light has illuminated before leaving the station;

- Initiate power - Drivers apply power as they start from a stop, or to increase train speed due to a change in signal aspect, line speed, or to maintain a speed profile;

- Change power - drivers change the power according to the train performance;

- Acknowledge vigilance alarm - drivers receive a buzzer warning at periodic intervals, which they must acknowledge by toggling a foot pedal (vigilance device; also known as dead man's pedal). If they do not respond within 7 seconds, the train emergency brake will be automatically applied.

The data therefore provides information on all routine actions performed by the driver to control the train and driver taskload can be calculated from this data by summing the number of actions within a set time period (e.g. the number of actions per minute). However, there are a number of driver tasks that are not logged in the data, particularly communications and passenger interactions (e.g. operating the passenger information system, responding to passenger queries, etc.). The OTDR also gives limited insight into the cognitive processing associated with the actions. It may still be useful for monitoring and comparing different journeys and different journey phases.

\section{Case Study Results}

Figure 3 shows an example histogram describing the number of actions in each minute of one of the train journeys analysed. The graph clearly shows variation in activity levels over the course of the journey, with a maximum of 19 and a minimum of 1 actions in each minute. 


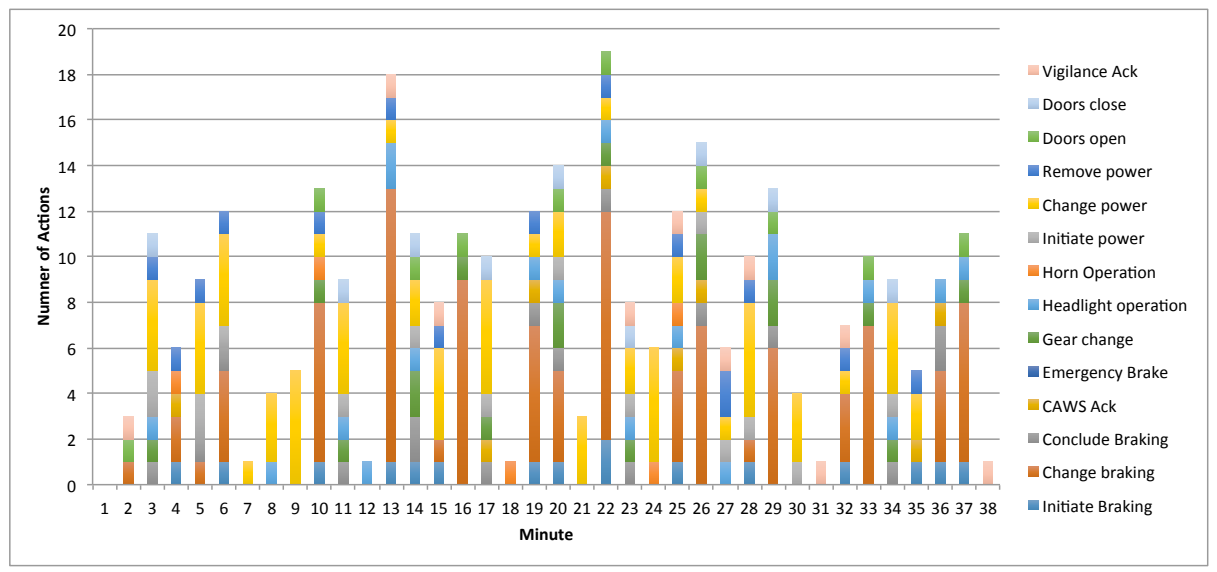

Figure 3: Train driver actions over a typical journey

The actions can be analysed in terms of journey phase, i.e. station duties, departing stations, arriving at stations, and travelling between stations. The journey stages were demarked according to the model described by Balfe $\&$ Smith [18]. Figure 4 shows a typical journey speed profile and describes the four main phases repeated throughout the journey.
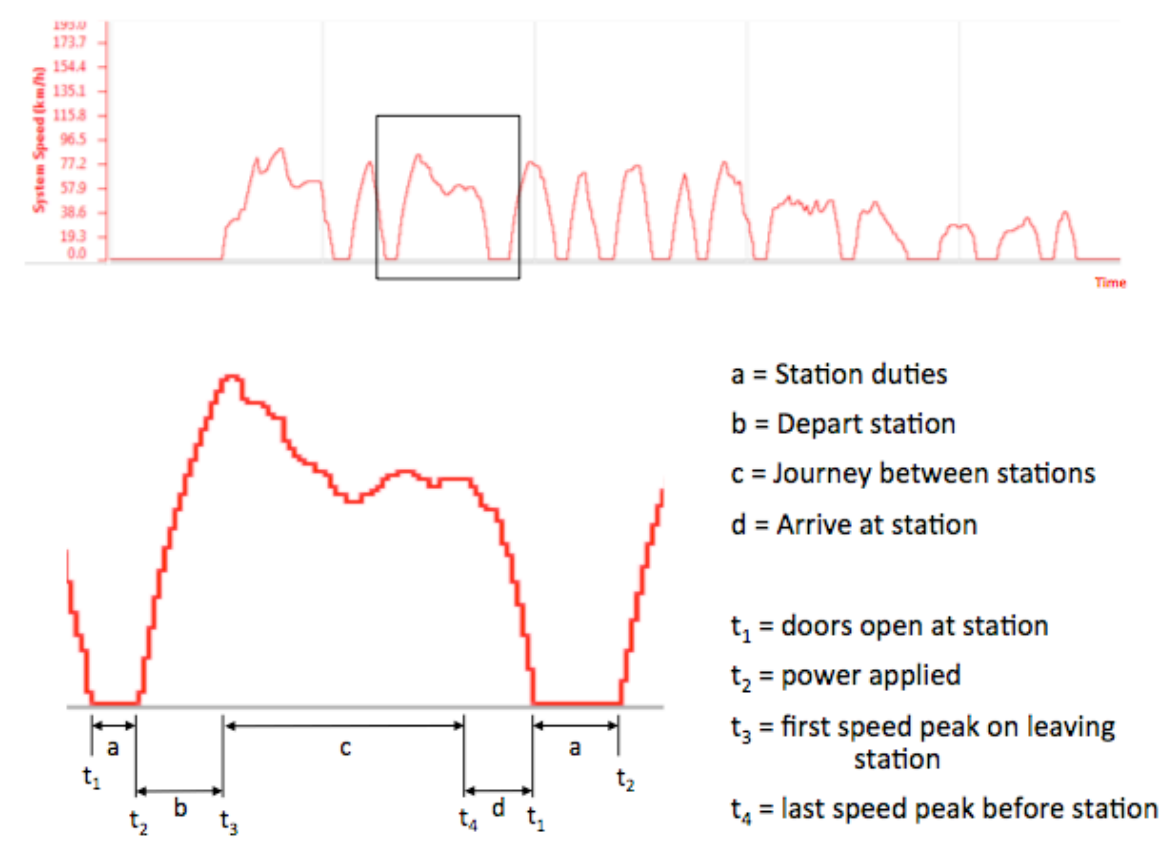

Figure 4: Train journey analysis framework (adapted from [18]) 
Figure 5 shows the mean number of actions per minute, and maximum and minimum number of actions, for each of the four journey stages across all 16 analysed journeys. The graph shows that the arrival at stations has the highest number of actions per minute, due to adjustments in braking levels approaching stations. Station duties and departing stations have similar action levels. It should be noted that the 16 journeys analysed were all commuter journeys, with relatively few instances of the 'between' stations stage and three journeys had no 'between' station stages at all. Between stations may be expected to generate relatively little task load as drivers simply maintain the required speed profile, however it can also involve stopping at red signals and this increases the actions required by the driver.

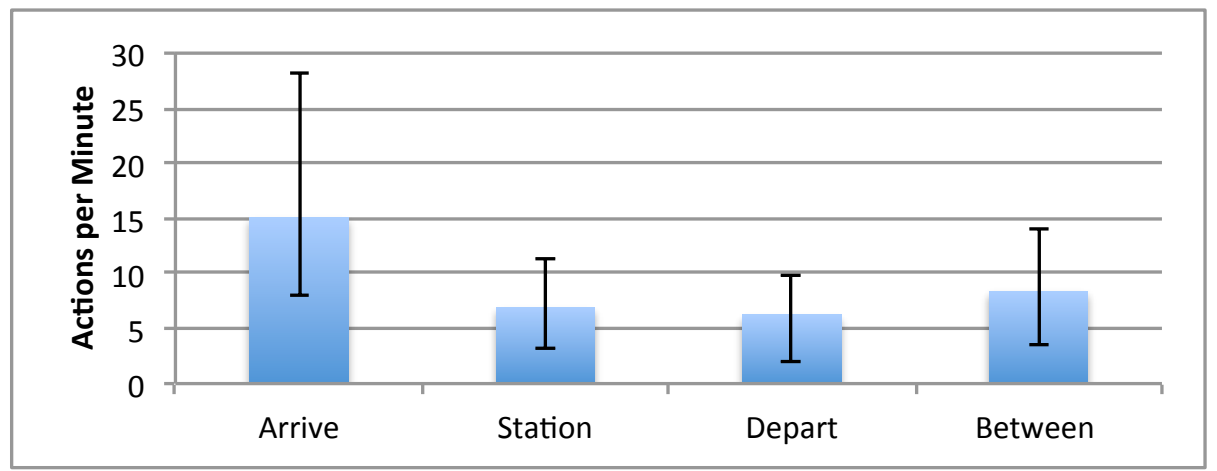

Figure 5: Average, maximum and minimum actions per minute for each of the four journey stages

Figure 6 shows a breakdown of the actions for each journey phase, shown as a percentage of the total actions in each phase. Braking, applying power and door operation are the dominant activities. As would be expected, a low level of braking actions are seen during station departures (comprising only 3\% of station departure actions). Drivers may apply the brakes when departing to test a train's braking characteristics, known as a running brake test. Similarly, power applications are rare during arrival phases ( $2 \%$ of arrival actions). Door operation is seen only in the station duties phase and the arrival phase, as on some occasions the doors were opened before the train had registered coming to a stop. Gear changes are also predominantly seen in the station phase, as drivers put the train in neutral after stopping in the station and replace the gear to forward when preparing to depart. Warning acknowledgements, headlight operations and horn operations were spread across all four phases. 


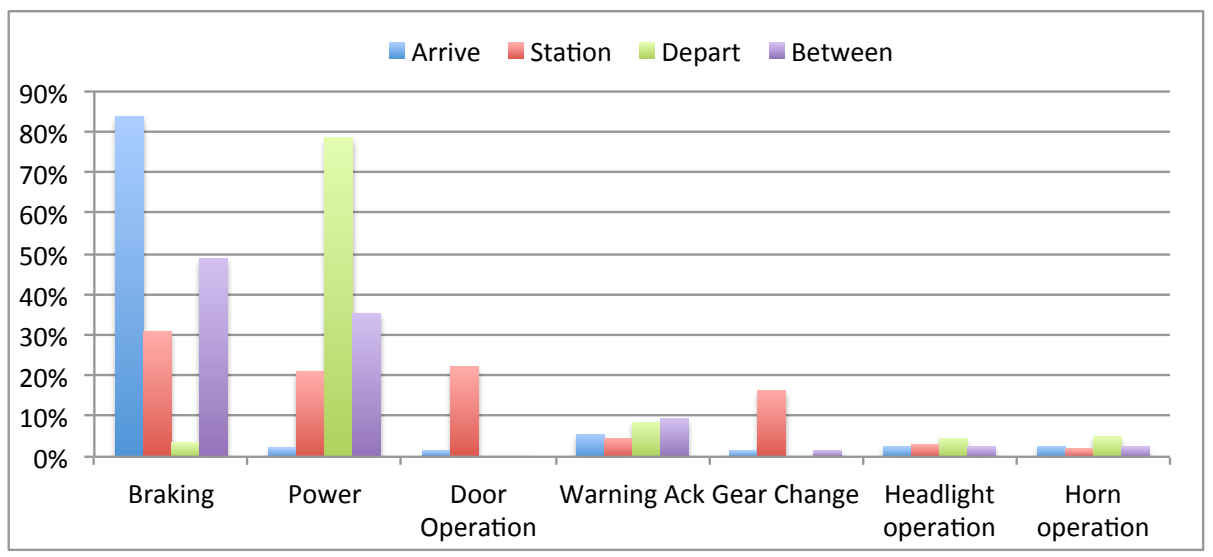

Figure 6: Proportion of actions of each of the four journey stages

\subsection{Weighted Method}

Since all actions are not equal in terms of the underlying cognitive processing, a more nuanced measurement of taskload could be constructed by weighting the different actions. A possible framework is described below as an illustration, although it should be noted that this framework is based only on preliminary task analyses, and has not been validated with train driving experts.

Table 2 shows the perception and memory activities associated with each of the physical actions logged in the OTDR. Actions with more cognitive activities associated with them can be assumed to place a higher load on the train driver. For example, initiating braking requires the driver to be aware of the location and speed of the train, drawing on route knowledge to determine the point at which to apply the brakes. The reason for the brake application may be to maintain line speed, a change of signal aspect, or to stop at a station. A more detailed framework could calculate individual factors for each of these events.

Table 2: Relative load for different actions

\begin{tabular}{|l|l|l|l|}
\hline Action & Perception & Memory & Relative load \\
\hline Initiate Braking & $\begin{array}{l}\text { Location } \\
\text { Speed } \\
\text { Signal Aspect }\end{array}$ & $\begin{array}{l}\text { Route knowledge } \\
\text { Signal aspect } \\
\text { Train characteristics }\end{array}$ & High \\
\hline Change Braking & Braking performance & Route knowledge & Medium \\
\hline $\begin{array}{l}\text { Conclude } \\
\text { Braking }\end{array}$ & $\begin{array}{l}\text { Speed } \\
\text { Signal Aspect }\end{array}$ & & Low \\
\hline $\begin{array}{l}\text { Acknowledge } \\
\text { CAWS }\end{array}$ & $\begin{array}{l}\text { Buzzer } \\
\text { Signal aspect }\end{array}$ & Signal aspect & Medium \\
\hline $\begin{array}{l}\text { Emergency } \\
\text { Brake }\end{array}$ & $\begin{array}{l}\text { Emergency situation } \\
\text { Error }\end{array}$ & Rules & High \\
\hline Gear Change & Doors closed & & Low \\
\hline
\end{tabular}




\begin{tabular}{|l|l|l|l|}
\hline & Signal upgrade & & \\
\hline $\begin{array}{l}\text { Headlight } \\
\text { operation }\end{array}$ & $\begin{array}{l}\text { Approaching train } \\
\text { Location }\end{array}$ & $\begin{array}{l}\text { Route knowledge } \\
\text { Rules }\end{array}$ & Medium \\
\hline Horn operation & $\begin{array}{l}\text { Sign } \\
\text { Location }\end{array}$ & Route knowledge & Medium \\
\hline Initiate power & $\begin{array}{l}\text { Station checks } \\
\text { Signal upgrade }\end{array}$ & $\begin{array}{l}\text { Rules } \\
\text { Signal aspect }\end{array}$ & High \\
\hline Change power & $\begin{array}{l}\text { Train performance } \\
\text { Speed }\end{array}$ & Route knowledge & Medium \\
\hline Remove power & Speed & Route Knowledge & Medium \\
\hline Doors open & $\begin{array}{l}\text { Train speed } \\
\text { Location }\end{array}$ & Low \\
\hline Doors close & Station checks & Rules & High \\
\hline $\begin{array}{l}\text { Vigilance } \\
\text { device }\end{array}$ & Buzzer & & Low \\
\hline
\end{tabular}

In this example, initiating braking, emergency brake application, initiating power and closing train doors are all tasks with relatively higher load than the others. This is because they draw more deeply on the drivers' memory and/or require more perception and analysis of the environment. Concluding braking, gear changes, opening doors, and responding to the vigilance device are listed as low in relation to other tasks, as they are all simple responses to a stimulus. Weighting coefficients can then be applied to the high, medium and low rated actions for better estimation of cognitive task load based on actions undertaken. For illustration purposes, actions in this dataset rated high were weighted five times those rated low, and medium were weighted three times those rated low. Further research would be required to determine the correct coefficients.

Figure 7 shows the average, maximum and minimum weighted actions per minute for the four journey stages. Braking is still the dominant activity in this weighted method, and the braking associated with arriving at stations is further highlighted in the weighted method. 


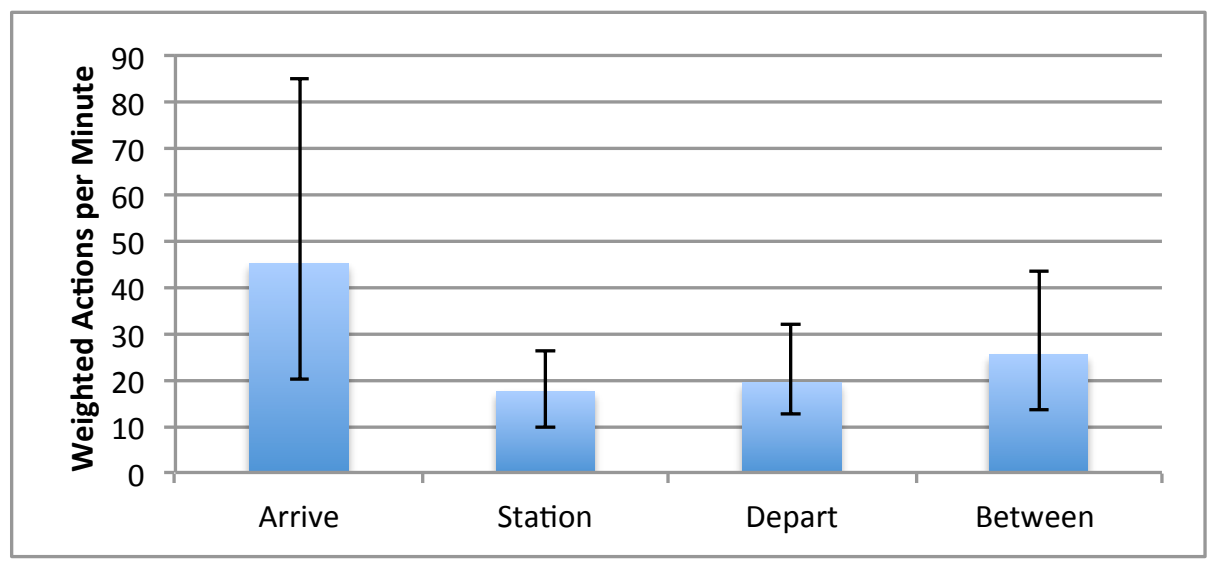

Figure 7: Weighted Average, Maximum and Minimum Actions per Minute

\section{Discussion and Conclusions}

This paper has presented a method for calculating train driver taskload from OTDR data, using transitions between states to infer driver actions. The results of the analysis of 16 journeys using the method illustrate the increase in taskload during arrival at stations, primarily due to continuous adjustment of train braking. Of course, the taskload is not the full characterisation of train driver workload, but it provides one piece of data that may be useful for monitoring driving performance, particularly in terms of underload. The method is completely unobtrusive, as it uses already existing data to construct the taskload model, and as such it may be a useful method of data collection in future studies involving train drivers.

In relation to the existing literature, the data presented here maps well to the cognitive task analysis undertaken by Hamilton and Clarke (2005b), although only the 'Drive Service' elements were presented in this paper. However, in terms of the models more based on human information processing, the OTDR data only provides detailed insight into 'actions' performed by the driver. Where signal aspect data is available from the OTDR, some small insight may be gained into perception particularly through analysis of reaction times to warnings of aspect changes. However this is very limited, and the wide range of other information used by drivers (e.g. landmarks, signals from platform staff, etc.) throughout journeys is not captured by the OTDR. Similarly, it is difficult to make any inferences on information analysis and driver decision-making from the data. The model of McLeod et al. (2005) describes the more contextual and cognitive processes that comprise train driving and highlights the limitations of taskload calculation alone for estimating driver workload. However, further analysis of large datasets from OTDR may provide some insight into the range of strategies used by different drivers in different situations.

Future research could apply the methodology to more journey types (particularly longer, intercity or high speed journeys) to compare key metrics with the shorter, 
commuter-type journeys analysed here. The example weighted method presented in this paper could also be further developed and the coefficients determined through structured manual observations of drivers and a comprehensive cognitive task analysis. They should also be validated with subject matter experts to ensure they accurately provide a more sensitive analysis of workload, as in Rizzo et al. and Rubio et al. $[19,20]$. The data collected in conjunction with the OTDR data described in this paper will also be analysed to determine whether there are any correlations between the OTDR taskload model and subjective or physiological measures of workload, providing some validation of the methodology.

\section{References}

1. Pickup, L., Wilson, J.R., Sharples, S., Norris, B., Clarke, T., Young, M.S. (2005). Fundamental examination of mental workload in the rail industry. Theoretical Issues in Ergonomics Science, 6(6), 463-482.

2. Naghiyev, A., Sharples, S., Ryan, B., Coplestone, A., Carey, M. (2016). Real workload verbal protocol data analysis of European Rail Traffic Management System train driving and conventional train driving. In 2016 IEEE International Conference on Intelligent Rail Transportation (ICIRT), pp. 191-196. IEEE

3. Luke, T., Brook-Carter, N., Parkes, A. M., Grimes, E., Mills, A. (2006). An investigation of train driver visual strategies. Cognition, Technology \& Work, 8(1), 15-29.

4. Branton, P. (1979). Investigations into the skills of train-driving. Ergonomics, 22 (2), 155164.

5. Doncaster, N. (2012). "By the seat of their pants" Cues and feedback used by train crew. In J.R. Wilson, A. Mills, T. Clarke, J. Rajan, \& N. Dadashi (Eds.) Rail Human Factors around the World: Impacts on and of People for Successful Rail Operations. Leiden: CRC Press, pp. 484-494.

6. Hamilton, W.I. \& Clarke, T. (2005a). Driver performance modelling and its practical application to railway safety. Applied Ergonomics, 36, 661-670.

7. Buksh, A., Sharples, S., Wilson, J.R., Coplestone, A., \& Morrisroe, G. (2013). A comparative cognitive task analysis of the different forms of driving in the UK rail system. In N. Dadashi, A. Scott, J.R. Wilson, \& A. Mills (Eds.) Rail Human Factors: Supporting Reliability, Safety, and Cost Reduction. London: Taylor \& Francis, pp. 173-182.

8. Zoer, I., Sluiter, J.K., Frings-Dresen, H.W. (2014). Psychological work characteristics, psychological workload and associated psychological and cognitive requirements of train driver. Ergonomics, 57(10), 1473-1487.

9. Gillis, I. (2007). Cognitive workload of train drivers. In J.R. Wilson, B. Norris, T. Clarke, \& A. Mills (Eds.) People and Rail Systems: Human Factors at the Heart of the Railway. Aldershot: Ashgate, pp. 91-101.

10. Hamilton, W.I. \& Clarke, T. (2005b). Driver performance modelling and its practical application to railway safety. In J.R. Wilson, B. Norris, T. Clarke, \& A. Mills (Eds.) Rail Human Factors: Supporting the Integrated Railway. Aldershot: Ashgate.

11. Naweed, A. (2014). Investigations into the skills of modern and traditional train driving. Applied Ergonomics, 45, 462-470.

12. Elliot, A.C., Garner, S.D., \& Grimes, E. (2007). The cognitive tasks of the driver: The approach and passage through diverging junctions. In J.R. Wilson, B. Norris, T. Clarke, \& A. Mills (Eds.) People and Rail Systems: Human Factors at the Heart of the Railway. Aldershot: Ashgate, pp. 115-123. 
13. Naweed, A., O'Keeffe, V., \& Tuckey, M.R. (2015). The art of train driving: Flexing the boundaries to manage risk within an inflexible system. Eat, Sleep, Work, 1.

14. McLeod, R.W., Walker, G.H., Moray, N. \& Mills, A. (2005). Analysing and modelling train driver performance. In J.R. Wilson, B. Norris, T. Clarke, \& A. Mills (Eds.) Rail Human Factors: Supporting the Integrated Railway. Aldershot: Ashgate.

15. Dunn, N., \& Williamson, A. (2012). Driving monotonous routes in a train simulator: The effect of task demand on driving performance and subjective experience. Ergonomics, 55(9), 997-1008.

16. Walker, G., \& Strathie, A. (2014) Combining human factors methods with transport data recordings. In N. Stanton, S. Landry, G. Di Bucchianic, \& A. Vallicelli (Eds.) Advances in Human Aspects of Transportation: Part 2.

17. Broekhoven, R. F. G. (2016). Comparison of real-time relative workload measurements in rail signallers. Twente: University of Twente.

18. Balfe, N., \& Smith, B. (2016). A framework for human factors analysis of railway on-train data. Paper presented at HFES-Europe Chapter Conference, Prague, October 2016.

19. Rizzo, L., Dondio, P., Delany, S.J., \& Longo, L. (2016) Modeling Mental Workload Via Rule-Based Expert System: A Comparison with NASA-TLX and Workload Profile. Artificial Intelligence Applications and Innovations, Volume 475 of the series IFIP Advances in Information and Communication Technology, pp 215-229.

20. Rubio, S., Díaz, E., Martín, J., \& Puente, J.M. (2004) Evaluation of subjective mental workload: A comparison of SWAT, NASA-TLX, and workload profile methods. Applied Psychology, 53(1), 61-86 